\title{
Nuclei and conuclei on Girard posets
}

\author{
David Kruml $^{a}$ and Jan Paseka ${ }^{a}$ \\ ${ }^{a}$ Department of Mathematics and Statistics, Faculty of Science, Masaryk University, \\ Kotlářská 2, CZ-611 37 Brno, Czech Republic, kruml,paseka@math.muni.cze-mail
}

\begin{abstract}
It is well-known that the semantics of a given fuzzy logic can be formally axiomatized by means of a residuated poset. Based on a notion of dualizing (cyclic) element we introduce the notion of a Frobenius (Girard) poset. With this paper we hope to contribute to the theory of Frobenius posets and Girard posets.

By means of a dualizing element we establish a one-to-one correspondence between a Frobenius poset and its opposite which is again a Frobenius poset. We also investigate some properties of nuclei and conuclei on Girard posets.

Finally, we discuss the relation between quantic nuclei and ideal conuclei on a Girard poset and its opposite. We show that they are in one-to-one correspondence.

Keywords: Residuated poset, Frobenius poset, Girard poset, Girard quantale, quantic nucleus, quantic conucleus, ideal conucleus.
\end{abstract}

\section{Introduction}

Residuation is one of fundamental concepts in ordered structures. Many interesting logics, such as Hájek's basic logic BL, developed in [5], and Esteva and Godo's monoidal t-norm based logic MTL, see [3], are prominent examples of formal systems of mathematical fuzzy logic. They arise as the logic of continuous and the logic of left-continuous t-norms respectively. It is also well-known that the semantics of such fuzzy logics can be formally axiomatized by means of residuated posets. Hence, residuated structures have been extensively studied for their importance in fuzzy logic and related areas.
It is important to remark that, on the object level, complete residuated posets are exactly quantales. The term quantale was suggested by Mulvey at the Oberwolfach Category Meeting [8] as "a quantization" of the term locale. Quantales are applied in linear [10] and other substructural logics and automaton theory [1]. Yetter [10] has shown that a non-commutative version of Girard's linear logic can be expressed in terms of quantales. The connection is given by the concept of dualizing element in a quantale.

The paper is organized as follows. First we present in Section 2 several necessary algebraic concepts as residuated poset, Frobenius poset and Girard poset. Motivated by the results on Girard quantales $[9,10]$ and dual quantales [6] (which are sometimes called Frobenius quantales [2]) we establish basic properties of Frobenius posets and Girard posets. The structures under investigation occur in the context of algebraic semantics of fuzzy logics.

In Section 3 we focus on the notions of quantic nuclei and conuclei on residuated posets and state their main properties. Some applications to Girard posets are addressed. In Section 4 we establish our main theorem that there is a one-to-one correspondence between quantic nuclei on a Girard poset and ideal conuclei on its opposite.

In this paper, we take for granted the concepts and results on quantales and residuated lattices. To obtain more information on these topics, we direct the reader to [4], [7] and [9]. To follow easily the arguments in the proofs we use the denotation $\stackrel{\text { (argument) }}{=}$, etc. to indicate which argument has been used in the respective step of the proof.

\section{Residuated posets, dualizing and cyclic elements}

In this section, we shall give the concept of a Frobenius poset and a Girard poset. By means of a dualizing el- 
ement we establish an one-to-correspondence between a Frobenius poset and its opposite.

Let $(P, \leq)$ be a partially ordered set. Its opposite is a partially ordered set $(P, \geq)$. An associative binary operation - on $(P, \leq)$ is said to be residuated if there exist binary operations $\leadsto$ and $\rightarrow$ on $P$ such that, for all $x, y, z \in P$,

$$
x \cdot y \leqslant z \Longleftrightarrow x \leqslant y \rightarrow z \Longleftrightarrow y \leqslant x \leadsto z .
$$

The operations $\leadsto$ and $\rightarrow$ are referred to as the right and left residual of ·, respectively. Then • is residuated if and only if it is order preserving in each argument and, for all $x, y, z \in P$, the inequality $x \cdot y \leqslant z$ has a largest solution for $x$ (namely $y \rightarrow z$ ) and for $y$ (namely $x \leadsto z)$. The residuals are uniquely determined by and $\leq$.

The system $\mathbf{P}=(P, \cdot, \leadsto, \rightarrow, \leqslant)$ is called a residuated partially ordered semigroup or residuated poset.

The following is well known (see [4]).

Proposition 2.1 Let $\mathbf{P}=(P, \cdot, \sim, \rightarrow, \leqslant)$ be a residuated poset. Then for all $x, y, z \in P$, we have

(r1) $y \leqslant z \Longrightarrow x \leadsto y \leqslant x \sim z ;$

(r2) $y \leqslant z \Longrightarrow z \leadsto x \leqslant y \leadsto x$;

(r3) $y \leqslant z \Longrightarrow x \rightarrow y \leqslant x \rightarrow z$;

(r4) $y \leqslant z \Longrightarrow z \rightarrow x \leqslant y \rightarrow x$;

$(\mathrm{r} 5) \quad x \leq(x \sim y) \rightarrow y, x \leq(x \rightarrow y) \leadsto y$;

(r6) $x \leadsto(y \rightarrow z)=y \rightarrow(x \leadsto z)$;

(r7) $x \rightarrow(y \rightarrow z)=(x \cdot y) \rightarrow z$;

(r8) $x \leadsto(y \sim z)=(y \cdot x) \leadsto z$;

$(\mathrm{r} 9) \quad x \leadsto y=((x \leadsto y) \rightarrow y) \leadsto y$;

$(\mathrm{r} 10) x \rightarrow y=((x \rightarrow y) \leadsto y) \rightarrow y$;

$(\mathrm{r} 11) y \rightarrow z \leqslant(x \rightarrow y) \rightarrow(x \rightarrow z)$;

$(\mathrm{r} 12) y \leadsto z \leqslant(x \leadsto y) \leadsto(x \leadsto z)$;

$(\mathrm{r} 13)$ If e is a unit of semigroup $(P, \cdot)$, then

$$
e \leq x \leadsto y \Longleftrightarrow x \leqslant y \Longleftrightarrow e \leqslant x \rightarrow y .
$$

An element $d$ of a residuated poset $\mathbf{P}$ is called a $d u a l$ izing element provided that

$$
(x \leadsto d) \rightarrow d=x=(x \rightarrow d) \leadsto d
$$

for all $x \in P$. An element $d \in P$ is called cyclic provided that

$$
x \rightarrow d=x \leadsto d
$$

for all $x \in P$. We shall denote $x \rightarrow d=x \leadsto d$ by $x^{\perp}$. Note that ${ }^{\perp}$ depends on the dualising element $d$. From now we assume that whenever we make use of ${ }^{\perp}$ that the dualising element $d$ is understood and fixed.

$\mathbf{P}$ is called a Frobenius poset (Girard poset) provided that it has a dualizing (cyclic and dualizing) element, respectively. Note that if $\mathbf{P}$ is a quantale then a Frobenius poset $\mathbf{P}$ is exactly a Frobenius quantale (see [2]) and a Girard poset $\mathbf{P}$ is exactly a Girard quantale.

Proposition 2.2 Let $\mathbf{P}$ be a Frobenius poset with a dualizing element $d$. Then for all $x, y \in P$, we have

(d1) $x \cdot(d \leadsto d) \leqslant y \Longleftrightarrow x \leqslant y$;

(d2) $(d \rightarrow d) \cdot x \leqslant y \Longleftrightarrow x \leqslant y$

(d3) $x \cdot(d \leadsto d)=x ;$

$(\mathrm{d} 4)(d \rightarrow d) \cdot x=x ;$

(d5) $d \rightarrow d=(d \rightarrow d) \cdot(d \leadsto d)=d \leadsto d$;

(d6) $d \rightarrow d$ is the unit of $(P, \cdot)$;

$(\mathrm{d} 7)(x \leadsto d) \rightarrow y=(y \rightarrow d) \leadsto x ;$

$(\mathrm{d} 8)(x \leadsto d) \rightarrow y=((x \leadsto d) \cdot(y \leadsto d)) \rightarrow d$;

(d9) $x \rightarrow y=(y \rightarrow d) \sim(x \rightarrow d)$;

$(\mathrm{d} 10) x \leadsto y=(y \leadsto d) \rightarrow(x \sim d)$.

Proof. (d1): We have

$$
\begin{aligned}
& x \cdot(d \leadsto d) \leqslant y \stackrel{(\partial)}{\Longleftrightarrow} x \cdot(d \leadsto d) \leqslant(y \rightarrow d) \leadsto d \\
& \stackrel{(\text { res })}{\Longleftrightarrow}(y \rightarrow d) \cdot x \cdot(d \leadsto d) \leqslant d \stackrel{(\text { res }),(\partial)}{\Longleftrightarrow} \\
& (y \rightarrow d) \cdot x \leqslant(d \leadsto d) \rightarrow d=d \stackrel{(\text { res })}{\Longleftrightarrow} \\
& (y \rightarrow d) \leqslant x \rightarrow d \stackrel{(\partial)}{\Longleftrightarrow} x \leqslant y .
\end{aligned}
$$

(d3): Since $x \leq x$ we have from $(\mathrm{d} 1)$ that $x \cdot(d \leadsto d) \leqslant$ $x$. Conversely, since $x \cdot(d \sim d) \leqslant x \cdot(d \leadsto d)$ we have again from $(\mathrm{d} 1)$ that $x \leqslant x \cdot(d \leadsto d)$.

(d2) and (d4): Similarly as (d1) and (d3).

(d5) and (d6): It follows immediately from (d3) and (d4).

(d7): We have

$$
\begin{aligned}
& z \leqslant(x \leadsto d) \rightarrow y \stackrel{(\text { res })}{\Longleftrightarrow} z \cdot(x \leadsto d) \leqslant y \stackrel{(\mathrm{r} 4)}{\Longleftrightarrow} \\
& y \rightarrow d \leqslant(z \cdot(x \leadsto d)) \rightarrow d \stackrel{(\mathrm{r} 7),(\partial)}{\Longleftrightarrow} \\
& y \rightarrow d \leqslant z \rightarrow((x \leadsto d) \rightarrow d)=z \rightarrow x \stackrel{\text { (res) }}{\Longleftrightarrow} \\
& (y \rightarrow d) \cdot z \leqslant x \stackrel{\text { (res) }}{\Longleftrightarrow} z \leqslant(y \rightarrow d) \leadsto x \text {. }
\end{aligned}
$$


(d8): We compute

$$
\begin{aligned}
((x \sim d) \cdot(y \leadsto d)) \rightarrow d \stackrel{(\mathrm{r} 7)}{=} \\
(x \leadsto d) \rightarrow((y \leadsto d) \rightarrow d) \stackrel{(\partial)}{=} \quad(x \leadsto d) \rightarrow y .
\end{aligned}
$$

(d9): We obtain

$$
\begin{aligned}
x \rightarrow y & \stackrel{(\partial)}{=}[(x \rightarrow d) \leadsto d] \rightarrow y \\
& \stackrel{(\mathrm{d} 7)}{=}(y \rightarrow d) \leadsto(x \rightarrow d) .
\end{aligned}
$$

(d10): Similarly as (d9).

Proposition 2.3 Let $\mathbf{P}$ be a Girard poset with a cyclic and dualizing element $d$. Then for all $x, y \in P$, we have

$$
\begin{aligned}
& \text { (g1) } x \rightarrow y=\left(x \cdot y^{\perp}\right)^{\perp} \text {; } \\
& \text { (g2) } x \leadsto y=\left(y^{\perp} \cdot x\right)^{\perp} .
\end{aligned}
$$

Proof. (g1): We have

$$
\begin{aligned}
z \leqslant x \rightarrow y & \stackrel{(\mathrm{d} 9)}{\Longleftrightarrow} z \leqslant y^{\perp} \leadsto x^{\perp} \stackrel{(\text { res })}{\Longleftrightarrow} y^{\perp} \cdot z \leqslant x^{\perp} \\
& \stackrel{(\text { res })}{\Longleftrightarrow} y^{\perp} \leqslant z \rightarrow x^{\perp} \stackrel{(\mathrm{d} 9)}{\Longleftrightarrow} y^{\perp} \leqslant x \leadsto z^{\perp} \\
& \stackrel{(\text { res })}{\Longleftrightarrow} x \cdot y^{\perp} \leqslant z^{\perp} \stackrel{(\partial),(\mathrm{d} 4)}{\Longleftrightarrow} z \leqslant\left(x \cdot y^{\perp}\right)^{\perp} .
\end{aligned}
$$

(g2): Similarly as (g1).

Let $\mathbf{P}$ be a Frobenius poset with a dualizing element d. We define a structure $\mathbf{P}^{\partial_{d}}=(P,+, \searrow, \nearrow, \geqslant)$ as follows:

$$
\begin{aligned}
x+y & =((x \leadsto d) \cdot(y \leadsto d)) \rightarrow d \\
x \searrow z & =z \cdot(x \leadsto d) \\
y \nearrow z & =(y \rightarrow d) \cdot z .
\end{aligned}
$$

Theorem 2.4 Let $\mathbf{P}$ be a Frobenius poset with a dualizing element d. Then $\mathbf{P}^{\partial_{d}}$ is a Frobenius poset with a dualizing element $d \rightarrow d$.

Proof. Let $x, y, z \in P$. We compute:

$$
\begin{aligned}
& x+y \geqslant z \stackrel{\left(\partial_{d}\right)}{\Longleftrightarrow}(x \leadsto d) \rightarrow y \geqslant z \stackrel{(\text { res })}{\Longleftrightarrow} \\
& y \geqslant z \cdot(x \leadsto d) \stackrel{(\mathrm{r} 4),(\partial)}{\Longleftrightarrow} y \rightarrow d \leqslant(z \cdot(x \leadsto d)) \rightarrow d \\
& \stackrel{(\mathrm{r} 7),(\partial)}{\Longleftrightarrow} y \rightarrow d \leqslant z \rightarrow((x \leadsto d) \rightarrow d)=z \rightarrow x \\
& \stackrel{\text { (res) }}{\Longleftrightarrow} x \geqslant(y \rightarrow d) \cdot z \stackrel{\left(\partial_{d}\right)}{\Longleftrightarrow} x \geqslant y \nearrow z \text {. }
\end{aligned}
$$

Similarly, we have

$$
\begin{aligned}
& x+y \geqslant z \stackrel{\left(\partial_{d}\right)}{\Longleftrightarrow}(y \rightarrow d) \leadsto x \geqslant z \stackrel{(\text { res })}{\Longleftrightarrow} \\
& x \geqslant(y \rightarrow d) \cdot z \stackrel{(\mathrm{r} 2),(\partial)}{\Longleftrightarrow} x \leadsto d \leqslant((y \rightarrow d) \cdot z) \leadsto d \\
& \stackrel{(\mathrm{r} 8),(\partial)}{\Longleftrightarrow} x \leadsto d \leqslant z \leadsto((y \rightarrow d) \leadsto d)=z \leadsto y \\
& \stackrel{(\text { res })}{\Longleftrightarrow} y \geqslant z \cdot(x \leadsto d) \stackrel{\left(\partial_{d}\right)}{\Longleftrightarrow} y \geqslant x \searrow z \text {. }
\end{aligned}
$$

The operation + is associative because

$$
\begin{aligned}
& (x+y)+z \stackrel{\left(\partial_{d}\right)}{=}(((x \leadsto d) \cdot(y \leadsto d)) \rightarrow d)+z \stackrel{\left(\partial_{d}\right)}{=} \\
& (((((x \leadsto d) \cdot(y \sim d)) \rightarrow d) \leadsto d) \cdot(z \leadsto d)) \rightarrow d \\
& \stackrel{(\partial)}{=}(((x \leadsto d) \cdot(y \leadsto d)) \cdot(z \leadsto d)) \rightarrow d \\
& \stackrel{\text { (assoc.) }}{=}((x \leadsto d) \cdot((y \leadsto d) \cdot(z \leadsto d))) \rightarrow d \stackrel{(\partial)}{=} \\
& ((((x \leadsto d) \cdot((y \leadsto d) \cdot(z \leadsto d))) \rightarrow d) \leadsto d) \rightarrow d \\
& \stackrel{(\mathrm{r} 7),}{=}\left(\partial_{d}\right)(((x \leadsto d) \rightarrow(y+z)) \leadsto d) \rightarrow d \\
& \stackrel{(\partial),(\mathrm{r} 7),(\partial)}{=}((x \leadsto d) \cdot((y+z) \leadsto d)) \rightarrow d \\
& \stackrel{\left(\partial_{d}\right)}{=} x+(y+z) \text {. }
\end{aligned}
$$

Let us check that $d \rightarrow d$ is a dualizing element of $\mathbf{P}^{\partial_{d}}$. We have

$$
\begin{aligned}
& (x \searrow(d \rightarrow d)) \nearrow(d \rightarrow d) \stackrel{\left(\partial_{d}\right)}{=} \\
& ((d \rightarrow d) \cdot(x \leadsto d)) \nearrow(d \rightarrow d) \stackrel{(\mathrm{d} 4)}{=} \\
& (x \leadsto d) \nearrow(d \rightarrow d) \stackrel{\left(\partial_{d}\right)}{=} \\
& ((x \leadsto d) \rightarrow d) \cdot(d \rightarrow d) \stackrel{(\partial),(\mathrm{d} 3)}{=} x .
\end{aligned}
$$

Similarly we obtain $(x \nearrow(d \rightarrow d)) \searrow(d \rightarrow d)=x$.

Moreover, $x+d=(x \leadsto d) \rightarrow d=x$ and $d+y=(y \rightarrow$ d) $\leadsto d=y$.

Corollary 2.5 Let $\mathbf{P}$ be a Frobenius poset with a dualizing element $d$. Then $\mathbf{P}=\left(\mathbf{P}^{\partial_{d}}\right)^{\partial_{d \rightarrow d}}$.

Proof. Let us denote the multiplication in $\left(\mathbf{P}^{\partial_{d}}\right)^{\partial_{d \rightarrow d}}$ by $\odot$ and let $x, y \in P$. It is enough to check that $d=(d \rightarrow d) \nearrow(d \rightarrow d)$ and $x \cdot y=x \odot y$ since the residuals are determined by the multiplication and the order. Let us compute:

$$
\begin{aligned}
x \odot y & \stackrel{\left(\partial_{d}\right)}{=}(x \searrow(d \rightarrow d)) \nearrow y \\
& \stackrel{\left(\partial_{d}\right)}{=}((d \rightarrow d) \cdot(x \leadsto d)) \nearrow y \\
& \stackrel{(\mathrm{d} 4)}{=}(x \leadsto d) \nearrow y \stackrel{\left(\partial_{d}\right)}{=}((x \leadsto d) \rightarrow d) \cdot y \\
& \stackrel{(\partial)}{=} x \cdot y .
\end{aligned}
$$

Similarly,

$$
\begin{gathered}
(d \rightarrow d) \nearrow(d \rightarrow d) \stackrel{(\mathrm{d} 5)}{=}(d \leadsto d) \nearrow(d \rightarrow d) \\
\left(\stackrel{\left(\partial_{d}\right)}{=}((d \sim d) \rightarrow d) \cdot(d \rightarrow d)\right. \\
(\partial), \stackrel{(\mathrm{d} 3, \mathrm{~d} 5)}{=} d .
\end{gathered}
$$

Corollary 2.6 Let $\mathbf{P}$ be a Girard poset with a dualizing and cyclic element d. Then $\mathbf{P}^{\partial_{d}}$ is a Girard poset with a dualizing and cyclic element $d \rightarrow d$. 
Proof. It is enough to check that $(d \rightarrow d)$ is cyclic in $\mathbf{P}^{\partial_{d}}$. Since $x \rightarrow d=x \leadsto d$ we can compute:

$$
\begin{aligned}
& x>(d \rightarrow d) \stackrel{\left(\partial_{d}\right)}{=}(x \rightarrow d) \cdot(d \rightarrow d) \\
&(\mathrm{d} 3, \mathrm{~d} 5)=(\text { clc }) \\
& \stackrel{(\mathrm{d} 4)}{=} x \rightarrow d \\
& \stackrel{=}{=}(d \rightarrow d) \cdot(x \leadsto d) \\
& \stackrel{\left(\partial_{d}\right)}{=} x \searrow(d \rightarrow d) .
\end{aligned}
$$

\section{Quantic nuclei and conuclei on residuated posets}

Quantic nuclei and quantic conuclei form an important method for constructing new quantales (see $[7,9]$ ). In this section, we shall introduce quantic nuclei, quantic conuclei and ideal conuclei on residuated posets and discuss their basic properties. It will be shown that the set of fixed points of such operators can be equipped with a structure of a residuated poset. En route, we show how to construct Girard posets from cyclic elements.

A quantic nucleus (conucleus) on a residuated poset $\mathbf{P}$ is a closure (coclosure) operator $j$ (i.e., orderpreserving, inflationary (decreasing) and idempotent), respectively, such that

$$
j(x) \cdot j(y) \leqslant j(x \cdot y)
$$

for all $x, y \in P$.

Reacall that an operator $j$ on a partially ordered set $(P, \leq)$ is called inflationary (decreasing) if $x \leq j(x)$ $(x \geq j(x))$ for all $x \in P$, respectively.

An ideal conucleus on $\mathbf{P}$ is a coclosure operator $j$ such that

$$
x \cdot j(y) \leqslant j(x \cdot y) \text { and } j(x) \cdot y \leqslant j(x \cdot y)
$$

for all $x, y \in P$. Clearly, any ideal conucleus is a quantic conucleus. Moreover, a closure operator $j$ on $\mathbf{P}$ is a quantic nucleus if and only if $x \cdot j(y) \leqslant j(x \cdot y)$ and $j(x) \cdot y \leqslant j(x \cdot y)$ for all $x, y \in P$. We denote by $N \mathbf{P}(C o N \mathbf{P}, I C N \mathbf{P})$ the poset of all quantic nuclei (conuclei, ideal conuclei) on $\mathbf{P}$ with order defined pointwise.

For any operator $j$ on a residuated poset $\mathbf{P}$ we denote by $P_{j}$ the set $\{x \in P \mid j(x)=x\}$.

Lemma 3.1 Let $\mathbf{P}$ be a residuated poset and $j$ a closure operator on $\mathbf{P}$. The following conditions are equivalent.

(i) $j$ is a quantic nucleus on $\mathbf{P}$ (ii) $x \leadsto j(y) \in P_{j}$ and $x \rightarrow j(y) \in P_{j}$, for all $x, y \in$ $P$.

(iii) $x \sim y \in P_{j}$ and $x \rightarrow y \in P_{j}$, for all $x \in P, y \in$ $P_{j}$.

Proof. (i) $\Longrightarrow$ (ii). Let $x, y \in P$. We compute:

$$
j(x \leadsto j(y)) \cdot x \stackrel{(\operatorname{lax})}{\leqslant} j((x \leadsto j(y)) \cdot x)^{(\text {res })} \leqslant j(y) .
$$

It follows

$$
x \sim j(y) \stackrel{(\text { clos })}{\leqslant} j(x \sim j(y)) \stackrel{(\text { res })}{\leqslant} x \leadsto j(y) .
$$

Hence $x \leadsto j(y)=j(x \leadsto j(y)) \in P_{j}$ and similarly $x \rightarrow j(y) \in P_{j}$.

(ii) $\Longrightarrow$ (iii). It is transparent.

(iii) $\Longrightarrow$ (i). Let $x, y \in P$. We have $x \cdot y^{(\operatorname{clos})} \leqslant(x \cdot y)$. We get

$$
\stackrel{(\mathrm{res})}{x \leqslant y \rightarrow j(x \cdot y) \stackrel{(\mathrm{iii})}{=}} j(y \rightarrow j(x \cdot y)) \in P_{j} .
$$

Hence $j(x) \stackrel{(\operatorname{clos})}{\leqslant} j(y \rightarrow j(x \cdot y))=y \rightarrow j(x \cdot y)$, i.e., (res)

$j(x) \cdot y \leqslant j(x \cdot y)$ and similarly $x \cdot j(y) \leqslant j(x \cdot y)$.

Let $\mathbf{P}$ be a residuated poset and $j$ a quantic nucleus on $\mathbf{P}$. We put, for all $x, y \in P_{j}$,

$$
x \cdot{ }_{j} y=j(x \cdot y) .
$$

Proposition 3.2 Let $\mathbf{P}$ be a residuated poset and $j$ a quantic nucleus on $\mathbf{P}$. Then the structure $\mathbf{P}_{j}=$ $\left(P_{j}, \cdot_{j}, \leadsto, \rightarrow, \leqslant\right)$ is a residuated poset. Moreover, if $\mathbf{P}$ is a (complete) lattice then $\mathbf{P}_{j}$ is a (complete) lattice.

Proof. It is enough to check that, for all $x, y, z \in P_{j}$,

$$
x \cdot{ }_{j} y \leqslant z \Longleftrightarrow x \leqslant y \rightarrow z \Longleftrightarrow y \leqslant x \leadsto z .
$$

\section{(clos)}

Assume that $x \cdot{ }_{j} y \leqslant z$. Then $x \cdot y \leqslant j(x \cdot y) \leqslant z$, i.e., $x \leqslant y \rightarrow z$. Conversely, assume that $x \leqslant y \rightarrow z$. We have $x \cdot y^{(\text {res })} z$, i.e., $x \cdot{ }_{j} y \stackrel{(\mathrm{qn})}{=} j(x \cdot y)^{(\text {(los })} \leqslant(z)^{(\text {(clos) }}=z$. Similarly we obtain that $x \cdot{ }_{j} y \leqslant z \Longleftrightarrow y \leqslant x \sim z$.

Assume that $\mathbf{P}$ is a lattice. Then, for all $x, y \in P_{j}$, clearly $x \wedge_{\mathbf{P}_{j}} y=x \wedge y$ and $x \vee_{\mathbf{P}_{j}} y=j(x \vee y)$ define lattice operations in $\mathbf{P}_{j}$. Similarly, if $\mathbf{P}$ is a complete lattice and $S \subseteq P_{j}$ then $\bigwedge_{\mathbf{P}_{j}} S=\bigwedge S$ and $\bigvee_{\mathbf{P}_{j}} S=$ $j(\bigvee S)$ define complete lattice operations in $\mathbf{P}_{j}$.

Lemma 3.3 Let $\mathbf{P}$ be a residuated poset and $j$ a coclosure operator on $\mathbf{P}$. The following conditions are equivalent. 
(i) $j$ is a quantic conucleus on $\mathbf{P}$.

(ii) $j(x) \cdot j(y) \in P_{j}$, for all $x, y \in P$.

(iii) $x \cdot y \in P_{j}$, for all $x, y \in P_{j}$.

Proof. (i) $\Longrightarrow$ (ii). Let $x, y \in P$. We compute:

$$
\begin{gathered}
j(x) \cdot j(y) \stackrel{\text { (cocl. op. })}{=} j(j(x)) \cdot j(j(y)) \stackrel{(\operatorname{lax})}{=} j(j(x) \cdot j(y)) \\
\stackrel{\text { (cocl. op. })}{\leqslant} j(x) \cdot j(y) .
\end{gathered}
$$

It follows that $j(x) \cdot j(y) \in P_{j}$.

(ii) $\Longrightarrow$ (iii). It is transparent.

(iii) $\Longrightarrow$ (i). Let $x, y \in P$. We have

$$
j(x \cdot y) \stackrel{\text { (cocl. op. })}{\geqslant} j(j(x) \cdot j(y)) \stackrel{(\mathrm{iii})}{=} j(x) \cdot j(y) .
$$

Let $\mathbf{P}$ be a residuated poset and $j$ a quantic conucleus on $\mathbf{P}$. We put, for all $x, y \in P_{j}$,

$$
x \leadsto_{j} y=j(x \leadsto y) \text { and } x \rightarrow_{j} y=j(x \rightarrow y) .
$$

Proposition 3.4 Let $\mathbf{P}$ be a residuated poset and $j$ a quantic conucleus on $\mathbf{P}$. Then the structure $\overline{\mathbf{P}}_{j}=$ $\left(P_{j}, \cdot, \sim_{j}, \rightarrow_{j}, \leqslant\right)$ is a residuated poset. Moreover, if $\mathbf{P}$ is a (complete) lattice then $\overline{\mathbf{P}}_{j}$ is a (complete) lattice.

Proof. It is enough to check that, for all $x, y, z \in P_{j}$,

$$
x \cdot y \leqslant z \Longleftrightarrow x \leqslant y \rightarrow_{j} z \Longleftrightarrow y \leqslant x \sim_{j} z .
$$

$$
\text { (res) }
$$

Assume that $x \cdot y \leqslant z$. Then $x \leqslant y \rightarrow z$, i.e.,

$$
x \stackrel{\text { (cocl. op. })}{\leqslant} j(x) \stackrel{\text { (cocl. op.) }}{\leqslant} j(y \rightarrow z) \stackrel{(\mathrm{qc})}{=} y \rightarrow_{j} z .
$$

Conversely, assume that $x \leqslant y \rightarrow_{j} z$. We have

$$
x \stackrel{\text { (cocl. op.) }}{=} j(x) \stackrel{(\mathrm{qc})}{\leqslant} j(x \rightarrow y) \stackrel{\text { (cocl. op.) }}{\leqslant} y \rightarrow z,
$$

(res)

i.e., $x \cdot y \leqslant z$. Similarly we obtain that $x \cdot y \leqslant z \Longleftrightarrow$ $y \leqslant x \sim_{j} z$.

Assume that $\mathbf{P}$ is a lattice. Then, for all $x, y \in P_{j}$, clearly $x \wedge \overline{\mathbf{P}}_{j} y=j(x \wedge y)$ and $x \vee \overline{\mathbf{P}}_{j} y=x \vee y$ define lattice operations in $\overline{\mathbf{P}}_{j}$. Similarly, if $\mathbf{P}$ is a complete lattice and $S \subseteq P_{j}$ then $\bigwedge_{\overline{\mathbf{P}}_{j}} S=j(\bigwedge S)$ and $\bigvee_{\overline{\mathbf{P}}_{j}} S=$ $\bigvee S$ define complete lattice operations in $\overline{\mathbf{P}}_{j}$.

Lemma 3.5 Let $\mathbf{P}$ be a residuated poset with a left (right) unit e and $g: P \rightarrow P$ a map. Then $g$ is an ideal conucleus if and only if

$$
\begin{aligned}
& g(x) \rightarrow g(y)=g(x) \rightarrow y \text { and } \\
& g(x) \leadsto g(y)=g(x) \leadsto y
\end{aligned}
$$

for all $x, y \in P$.
Proof. Assume that $g$ is an ideal conucleus and $x, y \in$

$P$. Since $g(y)^{\text {(cocl. op.) }} \leqslant y$ we have from (r3) and (r1) that $g(x) \rightarrow g(y) \leqslant g(x) \rightarrow y$ and $g(x) \leadsto g(y) \leqslant g(x) \sim$ $y$. Let $u \leqslant g(x) \rightarrow y$. Then $u \cdot g(x) \leqslant y$. It follows that

$$
u \cdot g(x) \stackrel{\text { (cocl. op.) }}{=} u \cdot g(g(x)) \stackrel{\text { (ilax) }}{\leqslant} g(u \cdot g(x)) \leqslant g(y),
$$

i.e., $u \leqslant g(x) \rightarrow g(y)$. Hence $g(x) \rightarrow g(y)=g(x) \rightarrow y$. Similarly, $g(x) \leadsto g(y)=g(x) \sim y$.

Conversely, assume that $g(x) \rightarrow g(y)=g(x) \rightarrow y$ and $g(x) \leadsto g(y)=g(x) \leadsto y$ for all $x, y \in P$. Then $e \cdot g(x) \leqslant g(x)$, i.e.

$$
e^{(\text {res })} \leqslant g(x) \rightarrow g(x) \stackrel{(\text { icon })}{=} g(x) \rightarrow x .
$$

Hence $g(x) \leq x$ and $g$ is decreasing. Since $g$ is decreasing we have also $g(g(x)) \leqslant g(x)$. From

$$
e \leq g(x) \rightarrow g(x) \stackrel{(\text { icon })}{=} g(x) \rightarrow g(g(x))
$$

$$
\text { (res) }
$$

we obtain that $g(x) \stackrel{\leqslant}{\leqslant} g(g(x))$, i.e., $g$ is idempotent. Let $x \leqslant y$. Then $e \cdot x=x \leqslant y$, i.e., since $g(x) \leq x$ we have

$$
e^{(\text {res })} \leqslant x \stackrel{(\text { r } 4)}{\leqslant} g(x) \rightarrow y \stackrel{(\text { icon })}{=} g(x) \rightarrow g(y),
$$

i.e. $g(x)=e \cdot g(x) \stackrel{\text { (res) }}{\leqslant} g(y)$ and $g$ is order-preserving. It remains to check the condition (ilax). Assume that $x, y \in P$. Since $g(y) \leqslant y$ we obtain that $x \cdot g(y) \leqslant x \cdot y$. Hence $x \stackrel{\text { (res) }}{\leqslant} g(y) \rightarrow(x \cdot y) \stackrel{(\text { icon })}{=} g(y) \rightarrow g(x \cdot y)$, i.e., $x \cdot g(y) \stackrel{(\text { res })}{\leqslant} g(x \cdot y)$. Similarly, $g(x) \cdot y \leqslant g(x \cdot y)$ and $g$ is an ideal conucleus.

The case when $e$ is a right unit follows by same considerations.

In what follows we show that, similarly to $[10$, Proposition 1.7], a Girard poset can be constructed from any residuated poset $\mathbf{P}$ containing a cyclic element $d \in P$ such that $(d \rightarrow d) \rightarrow d \leqslant d$.

Proposition 3.6 Let $\mathbf{P}$ be a residuated poset and $d$ a cyclic element of $\mathbf{P}$.

Then the map $j_{d}: P \rightarrow P$ given by $j_{d}(x)=(x \rightarrow d) \rightarrow$ $d$ is a quantic nucleus on $\mathbf{P}$. If $(d \rightarrow d) \rightarrow d \leqslant d$ then $d \in P_{j_{d}}$ and $\mathbf{P}_{j_{d}}$ is a Girard poset.

Proof. Since $d$ is cyclic we always have $x \rightarrow d=x \leadsto$ $d$. 
First, the map $j_{d}$ is order preserving since it is a composition of two antitone maps.

Second, $x \rightarrow d \leq x \rightarrow d$ implies $(x \rightarrow d) \cdot x \leq d$. We obtain

$$
x \stackrel{(\text { res })}{\leqslant}(x \rightarrow d) \sim d \stackrel{(\text { clc })}{=}(x \rightarrow d) \rightarrow d=j_{d}(x) .
$$

From Proposition 2.1 (r10) we have that $x \rightarrow d=$ $((x \rightarrow d) \rightarrow d) \rightarrow d=j_{d}(x) \rightarrow d$. It follows that $j_{d}(x)=j_{d}\left(j_{d}(x)\right)$, i.e. $j_{d}$ is a closure operator on $\mathbf{P}$.

Let us compute:

$$
\begin{aligned}
(y \cdot[y \sim(x \rightarrow d)]) \cdot[(x \rightarrow d) \rightarrow d] \stackrel{(\text { res })}{\leqslant} \\
(x \rightarrow d) \cdot[(x \rightarrow d) \rightarrow d] \stackrel{(\text { res })}{\leqslant} d .
\end{aligned}
$$

Since $\cdot$ is associative we have

$$
[y \leadsto(x \rightarrow d)] \cdot[(x \rightarrow d) \rightarrow d] \stackrel{(\text { res })}{\leqslant} y \rightarrow d .
$$

From $y \leadsto(x \rightarrow d)^{(\mathrm{r} 8),(\operatorname{clc})}(x \cdot y) \rightarrow d$ and the fact that

$$
\begin{aligned}
y \rightarrow d & \stackrel{(\operatorname{clos})}{\leqslant} j_{d}(y \rightarrow d)=((y \rightarrow d) \rightarrow d) \rightarrow d \\
& =j_{d}(y) \rightarrow d
\end{aligned}
$$

we obtain

$$
[(x \cdot y) \rightarrow d] \cdot j_{d}(x) \leqslant y \rightarrow d \leqslant j_{d}(y) \rightarrow d .
$$

Hence

$$
[(x \cdot y) \rightarrow d] \cdot j_{d}(x) \cdot j_{d}(y) \stackrel{(\text { res })}{\leqslant} d
$$

i.e.,

$$
j_{d}(x) \cdot j_{d}(y) \stackrel{(\text { res })}{\leqslant}[(x \cdot y) \rightarrow d] \rightarrow d=j_{d}(x \cdot y) .
$$

Let $(d \rightarrow d) \rightarrow d \leqslant d$. Clearly,

$$
d \stackrel{(\text { clos })}{\leqslant} j_{d}(d)=(d \rightarrow d) \rightarrow d \leqslant d
$$

i.e., $d \in P_{j_{d}}$. We have to check that $d$ is cyclic and dualizing in $\mathbf{P}_{j_{d}}$. Since $\rightarrow$ and $\leadsto$ in $\mathbf{P}$ coincide with the respective residuals in $\mathbf{P}_{j_{d}} d$ is cyclic in $\mathbf{P}_{j_{d}}$. Hence it is enough to check that $d$ is dualizing in $\mathbf{P}_{j_{d}}$. Assume that $x \in P_{j_{d}}$. We compute:

$$
(x \leadsto d) \rightarrow d \stackrel{(\text { clc })}{=}(x \rightarrow d) \rightarrow d=j_{d}(x)=x .
$$

and similarly we obtain that $(x \rightarrow d) \leadsto d=x$.

Corollary 3.7 Let $\mathbf{P}$ be a residuated poset with a left (right) unit $e$ and $d$ a cyclic element of $\mathbf{P}$. Then $\mathbf{P}_{j_{d}}$ is a Girard poset.
Proof. Assume that $e$ is a left unit of $\mathbf{P}$. For any $x \in P$ we have that $e \cdot x=x$. Let us check that $(d \rightarrow d) \rightarrow d \leqslant d$. We know that $e \leq d \rightarrow d$. Thus

$$
\begin{aligned}
j_{d}(d) & =(d \rightarrow d) \rightarrow d \stackrel{(\mathrm{cycl})}{=}(d \rightarrow d) \sim d \\
& =\bigvee\{x \in P \mid(d \rightarrow d) \cdot x \leq d\} \\
& \leq \bigvee\{x \in P \mid e \cdot x \leq d\}=d .
\end{aligned}
$$

Hence $\mathbf{P}_{j_{d}}$ is a Girard poset.

The case when $e$ is a right unit follows by same considerations.

\section{Quantic nuclei and conuclei on Girard posets}

In this section, we shall discuss the relations between quantic nuclei on a Girard poset $\mathbf{P}$ and ideal conuclei on its opposite $\mathbf{P}^{\partial_{d}}$. The primary objective is to establish one-to-one correspondence between $N \mathbf{P}$ and $I C N \mathbf{P}^{\partial_{d}}$.

Let $g$ be map on a Girard poset $\mathbf{P}$. We define a map $g^{*}$ on $P$ by

$$
g^{*}(x)=g\left(x^{\perp}\right)^{\perp} .
$$

Notice that $g^{* *}=g$.

Let $g, h$ be maps on $\mathbf{P}$, i.e., $g, h \in \mathbf{P}^{P}$. Since $\mathbf{P}^{\partial_{d}}$ and $\mathbf{P}$ have the same base sets we have also that $g, h$ are maps on $\mathbf{P}^{\partial_{d}}$, i.e., $g, h \in\left(\mathbf{P}^{\partial_{d}}\right)^{P}$. Then

$$
\begin{aligned}
g^{*} \geqslant_{\left(\mathbf{P}^{\partial_{d}}\right)^{P} h^{*}} & \Longleftrightarrow g^{*}(x) \geqslant_{\mathbf{P}^{\partial_{d}}} h^{*}(x) \text { for all } x \in P \\
& \Longleftrightarrow g\left(x^{\perp}\right)^{\perp} \leqslant_{\mathbf{P}} h\left(x^{\perp}\right)^{\perp} \text { for all } x \in P \\
& \Longleftrightarrow g\left(x^{\perp}\right) \geqslant_{\mathbf{P}} h\left(x^{\perp}\right) \text { for all } x \in P \\
& \Longleftrightarrow g \geqslant_{\mathbf{P}^{P}} h .
\end{aligned}
$$

We obtain the following.

Theorem 4.1 Let $\mathbf{P}$ be a Girard poset with a dualizing and cyclic element $d, g$ be a map on $P$. Then $g$ is a quantic nucleus on $\mathbf{P}$ if and only if $g^{*}$ is a quantic nucleus on $\mathbf{P}^{\partial_{d}}$.

Proof. Since $\left(\operatorname{id}_{P}\right)^{*}=\operatorname{id}_{P}$ we have that $g$ is inflationary on $\mathbf{P}$ if and only if $g^{*}$ is inflationary on $\mathbf{P}^{\partial_{d}}$.

Assume that $g$ is order-preserving. Let $x \leqslant y$. Then $y^{\perp} \leqslant x^{\perp}$. Hence $g\left(y^{\perp}\right) \leqslant g\left(x^{\perp}\right)$, i.e., $g^{*}(x)=$ $g\left(x^{\perp}\right)^{\perp} \leqslant g\left(y^{\perp}\right)^{\perp}=g^{*}(y)$.

Let $g$ be idempotent. We have $g^{*}\left(g^{*}(x)\right)=$ $g\left(g\left(x^{\perp}\right)^{\perp \perp}\right)^{\perp}=g\left(g\left(x^{\perp}\right)\right)^{\perp}=g\left(x^{\perp}\right)^{\perp}=g^{*}(x)$.

Suppose that $g(x) \cdot g(y) \leqslant g(x \cdot y)$ for all $x, y \in P$. Let us verify that $g^{*}(x)+g^{*}(y) \geqslant g^{*}(x+y)$ for all $x, y \in P$. We have

$$
\begin{array}{cll}
g^{*}(x)+g^{*}(y) & \stackrel{(*),\left(\partial_{d}\right)}{=} & \left(\left(g\left(x^{\perp}\right)^{\perp}\right)^{\perp} \cdot\left(g\left(y^{\perp}\right)^{\perp}\right)^{\perp}\right)^{\perp} \\
& \stackrel{(\partial)}{=} & \left(g\left(x^{\perp}\right) \cdot g\left(y^{\perp}\right)\right)^{\perp}
\end{array}
$$


and

$$
\begin{aligned}
& g^{*}(x+y) \quad \stackrel{\left(\partial_{d}\right),(*)}{=} \quad g\left(\left(\left(x^{\perp} \cdot y^{\perp}\right)^{\perp}\right)^{\perp}\right)^{\perp} \\
& \stackrel{(\partial)}{=} \quad g\left(x^{\perp} \cdot y^{\perp}\right)^{\perp} \text {. }
\end{aligned}
$$

Since $g\left(x^{\perp}\right) \cdot g\left(y^{\perp}\right) \leqslant g\left(x^{\perp} \cdot y^{\perp}\right)$ we obtain from $(\partial)$ and (r4) that $\left(g\left(x^{\perp}\right) \cdot g\left(y^{\perp}\right)\right)^{\perp} \geqslant g\left(x^{\perp} \cdot y^{\perp}\right)^{\perp}$. This is equivalent with

$$
g^{*}(x)+g^{*}(y) \geqslant g^{*}(x+y) .
$$

Since $g^{* *}=g$ we have that $g$ is a quantic nucleus on $\mathbf{P}$ if and only if $g^{*}$ is a quantic nucleus on $\mathbf{P}^{\partial_{d}}$.

In view of Theorem 4.1 we have the following corollary.

Corollary 4.2 Let $\mathbf{P}$ be a Girard poset with a dualizing and cyclic element $d$. Then the posets NP and $N \mathbf{P}^{\partial_{d}}$ are order-isomorphic via the map ${ }^{*}$.

The following theorem generalizes [6, Theorem 3.6] and provides an important description of the relations between quantic nuclei and ideal conuclei on Girard posets.

Theorem 4.3 Let $\mathbf{P}$ be a Girard poset with a dualizing and cyclic element $d, g$ be a map on $P$. Then $g$ is a quantic nucleus on $\mathbf{P}$ if and only if $g^{*}$ is an ideal conucleus on $\mathbf{P}$.

Proof. By the same arguments as in Theorem 4.1 we obtain that $g$ is a closure operator on $\mathbf{P}$ if and only if $g^{*}$ is a coclosure operator on $\mathbf{P}$. Assume first that $g$ is a quantic nucleus. Let us check that $x \cdot g^{*}(y) \leqslant g^{*}(x \cdot y)$ (the case $g^{*}(x) \cdot y \leq g^{*}(x \cdot y)$ can be shown similarly). Since $g$ is a quantic nucleus we have

$$
\begin{aligned}
x \cdot g\left(x \rightarrow y^{\perp}\right) & \stackrel{\text { (clos. op. })}{\leqslant} g(x) \cdot g\left(x \rightarrow y^{\perp}\right) \\
& \stackrel{(\operatorname{lax})}{\leqslant} g\left(x \cdot\left(x \rightarrow y^{\perp}\right)\right) \stackrel{(\text { res })}{\leqslant} g\left(y^{\perp}\right) .
\end{aligned}
$$

From (g1) we obtain that $(x \cdot y)^{\perp}=x \rightarrow y^{\perp}$. Hence

$$
g\left((x \cdot y)^{\perp}\right) \leqslant x \leadsto g\left(y^{\perp}\right) \stackrel{(\mathrm{d} 10)}{=} g\left(y^{\perp}\right)^{\perp} \rightarrow x^{\perp} .
$$

It follows that $x \cdot g\left(y^{\perp}\right)^{\perp} \cdot g\left((x \cdot y)^{\perp}\right) \leqslant d$. This yields $x \cdot g\left(y^{\perp}\right)^{\perp} \leqslant g\left((x \cdot y)^{\perp}\right)^{\perp}$.

Therefore $x \cdot g^{*}(y) \leqslant g^{*}(x \cdot y)$.

Assume now that $g^{*}$ is an ideal conucleus. Since $\mathbf{P}$ has a unit $d \rightarrow d$ we compute (using twice Lemma 3.5):

$$
\begin{aligned}
& g(x) \cdot g(y) \leqslant g(x \cdot y) \stackrel{(\text { res })}{\Longleftrightarrow} \\
& g(x) \leqslant g(y) \rightarrow g(x \cdot y) \stackrel{(\mathrm{d} 9)}{=} g(x \cdot y)^{\perp} \leadsto g(y)^{\perp} \\
& \stackrel{(*)}{\Longleftrightarrow} g(x) \leqslant g^{*}\left((x \cdot y)^{\perp}\right) \leadsto g^{*}\left(y^{\perp}\right) \stackrel{\text { L. } 3.5}{\Longleftrightarrow} \\
& g(x) \leqslant g^{*}\left((x \cdot y)^{\perp}\right) \leadsto y^{\perp} \stackrel{(\text { res }),(\mathrm{d} 9)}{\Longleftrightarrow} \\
& g^{*}\left((x \cdot y)^{\perp}\right) \leqslant g(x) \rightarrow y^{\perp}=y \sim g(x)^{\perp}
\end{aligned}
$$

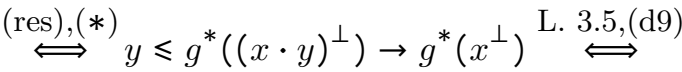

$$
\begin{aligned}
& y \leqslant g^{*}\left((x \cdot y)^{\perp}\right) \rightarrow x^{\perp}=x \sim g(x \cdot y) \\
& \stackrel{(\text { res })}{\Longleftrightarrow} x \cdot y \leqslant g(x \cdot y) \text {. }
\end{aligned}
$$

Since $g$ is inflationary, i.e., $x \cdot y \leqslant g(x \cdot y)$ we obtain that $g(x) \cdot g(y) \leqslant g(x \cdot y)$, i.e., $g$ is a quantic nucleus.

Consequently, we have the following result.

Corollary 4.4 Let $\mathbf{P}$ be a Girard poset with a dualizing and cyclic element $d$. Then the posets NP and $(I C N \mathbf{P})^{\text {op }}$ are order-isomorphic via the map ${ }^{*}$.

In the following, we establish a one-to-one correspondence between quantic nuclei and ideal conuclei on Girard posets.

Theorem 4.5 Let $\mathbf{P}$ be a Girard poset with a dualizing and cyclic element $d$. Then we have a series of order-isomorphisms

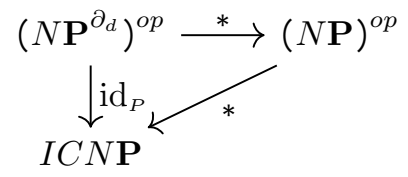

Proof. It follows immediately from Corollaries 4.2 and 4.4 , and the fact that ${ }^{*}{ }^{*}{ }^{*}=\operatorname{id}_{P}$.

Corollary 4.6 Let $\mathbf{P}$ be a Girard poset with a dualizing and cyclic element $d$. Then quantic nuclei on $\mathbf{P}^{\partial_{d}}$ coincide with ideal conuclei on $\mathbf{P}$ and quantic nuclei on $\mathbf{P}$ coincide with ideal conuclei on $\mathbf{P}^{\partial_{d}}$.

\section{Acknowledgement}

Research of the first author was supported by the project New approaches to aggregation operators in analysis and processing of data, Nr. 18-06915S by Czech Grant Agency (GAČR). Research of the second (corresponding) author was supported by the project Group Techniques and Quantum Information, Nr. MUNI/G/1211/2017 by Masaryk University Grant Agency (GAMU).

We thank the anonymous referees for the very thorough reading and contributions to improve our presentation of the paper. 


\section{References}

[1] S. Abramsky, S. Vickers, Quantales, observational logic and process semantics, Mathematical Structures in Computer Science 3 (1993) 161-227.

[2] P. Eklund, J. García, U. Höhle, J. Kortelainen, Semigroups in Complete Lattices: Quantales, Modules and Related Topics, Developments in Mathematics 54, Springer, Cham, 2018.

[3] F. Esteva, L. Godo, Monoidal t-norm logic: towards a logic for left-continuous t-norms, Fuzzy Sets and Systems 124 (3) (2001) 271-288.

[4] N. Galatos, P. Jipsen, C. Tsinakis, H. Ono, Residuated lattices: an algebraic glimpse at substructural logics, Studies in Logics and the Foundations of Mathematics 151, Elsevier, Oxford, 2007.

[5] P. Hájek, Metamathematics of Fuzzy Logic, Vol. 4 of Trends in Logic, Kluwer Academic Publishers, Dordrecht, 1998.

[6] S. Han, B. Zhao, Nuclei and conuclei on residuated lattices, Fuzzy Sets and Systems 172 (1) (2011) 51-70.

[7] D. Kruml, J. Paseka, Algebraic and categorical aspects of quantales, in: M. Hazewinkel (Ed.), Handbook of Algebra, Vol. 5, North-Holland, Amsterdam, 2008, pp. 323-362.

[8] C. J. Mulvey, \&, Suppl. Rend. Circ. Mat. Palermo, ser. II 12 (1986) 99-104.

[9] K. J. Rosenthal, Quantales and their applications, Pitman Research Notes in Mathematics Series 234, Longman Scientific \& Technical, Essex, 1990.

[10] D. Yetter, Quantales and (noncommutative) linear logic, Journal of Symbolic Logic 55 (1990) 338-353. 